\title{
Chloroacetate Esterase Staining Method
}

National Cancer Institute

\section{Source}

National Cancer Institute. Chloroacetate Esterase Staining Method. NCI Thesaurus. Code C28409.

Chloroacetate esterase (CAE) is a cytochemical staining technique to specifically identify cells of the granulocyte lineage, from the early promyelocyte stage to mature neutrophils. 